Revista Perspectivas Online: Exatas \& Engenharia Dezembro/2021, v.11, n.34, p. 44- 58 ISSN: 2236-885X (Online)

DOI: $10.25242 / 885 X 113420212476$

\title{
SIMULAÇÃO COMPUTACIONAL DO PRIMEIRO ESTÁGIO DE COMPRESSÃO DE UMA TURBINA A GÁS SOB REGIME TRANSIENTE
}

\author{
Matheus Mattos Pereira ${ }^{1}$; Silvio Eduardo Teixeira Pinto da Silva ${ }^{{ }^{*}}$ \& Patrick Silva Oliveira ${ }^{1}$
}

\section{RESUMO}

PEREIRA, M. M.; SILVA, S.E.T.P.; OLIVEIRA, P.S. Simulação computacional do primeiro estágio de compressão de uma turbina a gás sob regime transiente. Perspectivas Online: Exatas \& Engenharia, v.11, n.34, p.44-58, 2021.

O presente trabalho objetiva o estudo do funcionamento de um compressor axial, equipamento que tem como intuito a elevação de pressão do gás. Este estudo está direcionado a monitorar propriedades como vazão mássica, temperatura e pressão do ar, durante o processo de compressão do primeiro estágio, aplicando o Software Ansys Fluent. Para análise em questão, foram utilizadosf métodos computacionais, que operam através de resoluções de equações de Euler, de forma mais precisa e refinada. O controle dessas propriedades através de ferramentas da fluidodinâmica computacional, requer conhecimentos básicos sobre as principais equações presentes no processo de escoamento compressível, como também de todo processo necessário para criação da geometria e malha do compressor. Para obtenção de resultados finais mais próximos da realidade, o fluido durante seu processo de compressão seguiu condições de contorno e limites periódicos, configurada a geometria do equipamento no Software. Diante disso, foi constatado através dos valores das propriedades entre entrada e saída do compressor, que houve elevação de pressão, e variação do balanço de massa, pois o fluido se comportou de forma instável, concluindo assim que a simulação computacional consegue descrever o processo de compressão do ar como regime time periódico.

Palavras-chave: Compressor; Simulação; Regime Transiente;

${ }^{1}$ Institutos Superiores de Ensino do CENSA - ISECENSA - Laboratório de Análise e Projeto de Sistemas Mecânicos LAPSIM/ISECENSA, - Rua Salvador Correa, 139, Centro, Campos dos Goytacazes, RJ, CEP: 28035-310, Brasil.

(*) e-mail: silvioeduardo2@gmail.com

Data de recebimento: 09/11/2021. Aceito para publicação: 13/12/2021. Data de publicação: 21/12/2021. 
Revista Perspectivas Online: Exatas \& Engenharia Dezembro/2021, v.11, n.34, p. 44- 58

ISSN: 2236-885X (Online)

DOI: $10.25242 / 885 X 113420212476$

\title{
COMPUTATIONAL SIMULATION OF THE FIRST COMPRESSION STAGE OF A GAS TURBINE UNDER TRANSIENT REGIME
}

\author{
Matheus Mattos Pereira ${ }^{1}$; Silvio Eduardo Teixeira Pinto da Silva ${ }^{{ }^{*}}$ \& Patrick Silva Oliveira ${ }^{1}$
}

\begin{abstract}
PEREIRA, M. M.; SILVA, S.E.T.P.; OLIVEIRA, P.S. Computational simulation of the first compression stage of a gas turbine under transiente regime. Perspectivas Online: Exatas \& Engenharia, v.11, n.34, p.44-58, 2021.

The present work aims to study the operation of an axial compressor, equipment that aims to increase gas pressure. This study is aimed at monitoring properties such as mass flow, temperature and air pressure, during the first stage compression process, using Ansys Fluent Software. For the analysis in question, computational methods were used, which operate through the resolution of Euler's equations, in a more precise and refined way. The control of these properties through computational fluid dynamics tools requires basic knowledge about the main equations present in the compressible

necessary to create the geometry and mesh of the compressor. To obtain final results closer to reality, the fluid during its compression process followed boundary conditions and periodic limits, configured the equipment geometry in the Software. Therefore, it was verified through the values of the properties between compressor input and output, that there was a pressure increase, and mass balance variation, as the fluid behaved in an unstable way, thus concluding that the computer simulation is able to describe the process of air compression as a periodic time
\end{abstract} flow process, as well as the entire process

Keywords: Journal; Guidelines; Instructions.

\footnotetext{
${ }^{1}$ Institutos Superiores de Ensino do CENSA - ISECENSA - Laboratório de Análise e Projeto de Sistemas Mecânicos LAPSIM/ISECENSA, - Rua Salvador Correa, 139, Centro, Campos dos Goytacazes, RJ, CEP: 28035-310, Brasil; (*) e-mail: silvioeduardo2@gmail.com

Received: 09/11/2021. Accepted:.13/12/2021 Published online: 21/12/2021.
} 


\section{INTRODUÇÃO}

Os compressores são máquinas de fluxo responsável pelo aumento de pressão em um gás (normalmente ar), até o valor desejado, por meio de trabalho mecânico. Suas características principais são a capacidade de operar com vazões elevadas e também sua alta eficiência termodinâmica. Suas aplicações na indústria são na compressão de ar para a câmara de combustão em turbinas a gás, ou turbinas para aeronaves comerciais ou militares e sopradores de ar na indústria de petróleo e gás, petroquímica e siderúrgica (Simões, 2009).

Atualmente, os métodos computacionais conseguem controlar e solucionar diversas adversidades de trabalho que através de cálculos analíticos seriam impossíveis. Desta forma as ferramentas de simulação de escoamento, de análise de estrutura, e outras diversas aplicações, conseguem resolver situações especificas para realidade de várias aplicações industriais.

A simulação computacional utilizando Computational Fluid Dynamics, é baseada em resoluções de equações de Navier-Stokes através da aplicação de métodos de volumes finitos, possibilitando a redução de custos, maior praticidade, aumento na eficiência e menor probabilidade de acidentes, ocasionados por problemas funcionais de equipamentos considerados essenciais nos compressores.

O Objetivo deste trabalho é a avaliação da taxa de compressão no primeiro estágio de um compressor axial contendo 16 lâminas no rotor e 32 lâminas no estator. Além da taxa de compressão, a característica do escoamento também deve ser verificada e para isso será utilizado o conceito de uma modelagem de malha deslizante.

\section{METODOLOGIA}

No geral, quase todos os softwares de simulação numérica adotam o fluxograma presente na Figura 1 para o estudo ou solução numérica, sendo as etapas: os dados do problema, geometria, malha, condição de contorno, simulação e pós-processamento.

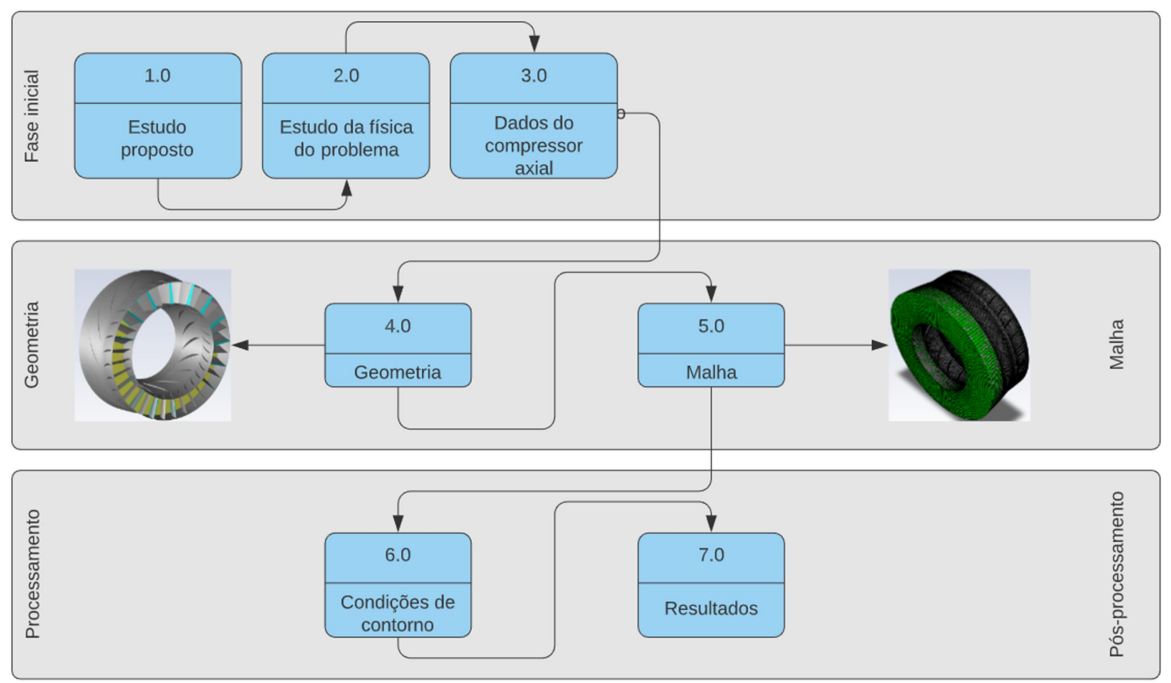

Figura 1: Fluxograma de Processo para execução de uma simulação numérica envolvendo CFD. 
Para o presente trabalho o conjunto de softwares utilizados foi o (C) 2020 ANSYS, Inc. SpaceClaim R2 (geometria), ICEM CFD R1 (malha), Fluent R2 (solver) para solução das equações de Euler para um fluido invíscido através das condições de contorno e métodos adotados e o pós-processamento das imagens e gráficos.

A construção da geometria utiliza um modelo de um estágio único de um compressor axial compreendido por duas linhas de lâminas (blades). A primeira fileira de lâmina é o rotor com um total de 16 lâminas das quais opera em velocidade rotacional de $37.500 \mathrm{rpm}$. A segunda fileira de lâminas é o estator que possui um total de 32 lâminas. As lâminas são posicionadas de tal forma que o domínio é rotacional e periódico. Além disso, o ângulo para os limites periódicos possui 22.5 graus. A Figura 2 apresenta os detalhes da geometria e as condições de contorno nomeadas para cada parte no CAD (Computer-Aided Design).

Devido ao alto número de Reynolds a malha pode ser tratada de forma menos refinada em ambas as lâminas, pois a análise baseia se na solução das equações de Euler utilizando modelo de turbulência configurado para fluido invíscido.

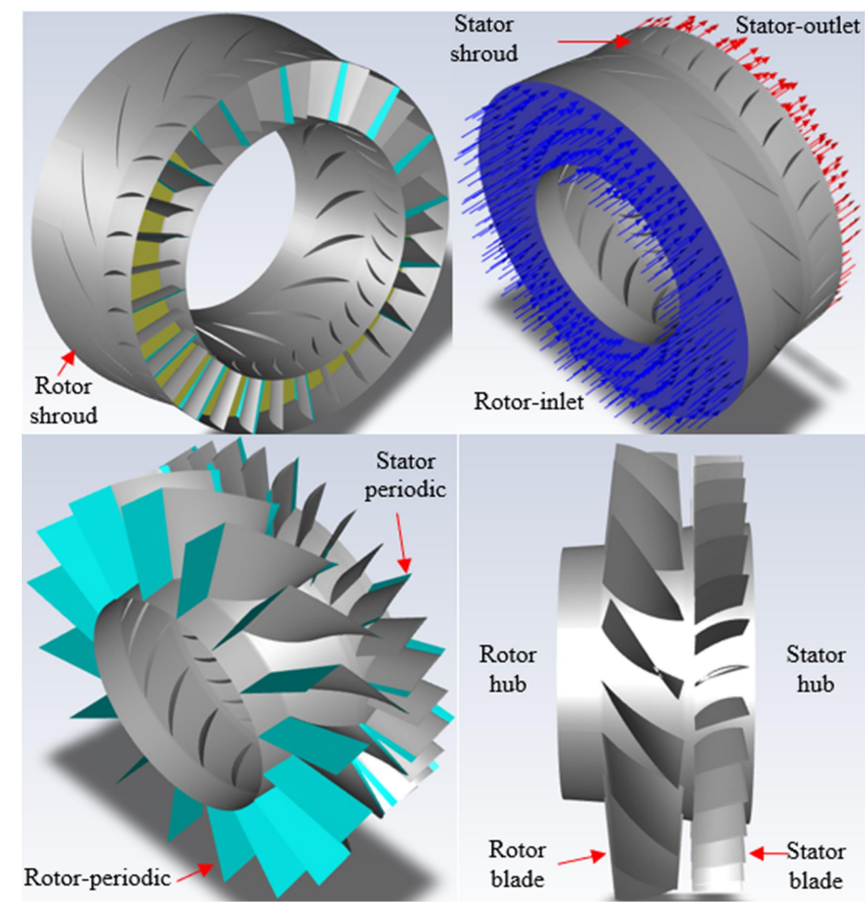

Figura 2: Detalhes da geometria do compressor axial.

A geometria foi construída para fins de pesquisa educacional e foi desenvolvida para rodar em solver acadêmicos, ou seja, com limitações no número de elementos de malha. A geometria final possui diâmetro interno/externo localizado no rotor-hub equivalente a 111,76 / 208,28mm. Possui diâmetro interno / externo localizado no stator-hub equivalente a 136,6 / $193,01 \mathrm{~mm}$. O comprimento total medindo do lado rotor-inlet até o stator-outlet é de $82 \mathrm{~mm}$.

As condições de contorno utilizadas em uma simulação estão relacionadas diretamente aos valores utilizados na inicialização, ou seja, uma equação diferencial com suas restrições localizadas, designadas como condição de contorno. Para que a solução tenha representação física correta e os resultados obtidos se aproximem da solução analítica/experimento é 
importante adotar condições de contorno corretas para solução do problema proposto, pois, para cada entrada do problema existe uma solução única extremamente dependente desta entrada.

Para o presente trabalho foram utilizadas cinco condições de contorno, sendo elas:

- Wall - A condição de parede foi definida para uma condição de fluido invíscido, considerando que a condição de velocidade do fluxo na parede deve ser diferente de zero, ou seja, não existirá fricção para promover aderência à superfície, portanto, na superfície o fluxo deve ser tangente a mesma e de acordo com a condição $\vec{V} * \vec{n}=0$ na superfície.

- Periodic - quando dois limites opostos estão conectados e seus valores são análogos ou o campo de fluxo e geometria permite movimentos rotacionais ou translacionais, esta solução deve ser utilizada.

Desta forma, para uma condição de contorno periódica com periodicidade rotacional, tem-se:

- Variação de pressão igual a zero através do plano periódico. Eixo de rotação deve ser definido na zona da célula fluida.

- Interface - A utilização de uma condição de limite para as interfaces define as regiões entre rotor e estator, é de fundamental importância sua configuração, pois, caso não configurada corretamente, permanecerá com uma condição de parede acarretando erros para solução.

- Pressure-inlet - Condição de contorno utilizada para definição da pressão total e temperatura total de entrada. Para escoamentos compressíveis esta equação é válida:

$$
\begin{aligned}
\frac{P_{\text {total }}}{P_{\text {static }}} & =\left(1+\frac{k-1}{2} M^{2}\right)^{\frac{k}{k-1}} \\
\frac{T_{t}}{T} & =1+\frac{k-1}{2} M^{2}
\end{aligned}
$$

Onde:

$P_{\text {total }}:$ Pressão total;

$P_{\text {static }}:$ Pressão estática;

$k$ : Coeficiente isentrópico;

$M:$ Número de mach;

$T_{t}:$ Temperatura total;

$T$ : Temperatura estática. 
- Pressure - outlet - Condição de contorno utilizada para definição da pressão total de saída.

A Tabela 1 apresenta as condições de contorno utilizadas no presente trabalho. A solução foi inicializada no Fluent utilizando velocidade absoluta para condição de contorno rotor-inlet com pressão estática de 0,9 atm $(91192,5 \mathrm{~Pa})$, velocidade de $130,9 \mathrm{~m} / \mathrm{s}$ e temperatura de $279,47 \mathrm{~K}$. Estes valores podem ser verificados a partir das equações para condição de contorno de pressão e temperatura na entrada conforme apresentado.

$$
\begin{gathered}
\frac{101325}{91192,5}=\left(1+\frac{1,4-1}{2} M^{2}\right)^{\frac{1,4}{1,4-1}} \rightarrow M=0,39087 \\
\frac{288}{T}=1+\frac{1,4-1}{2} 0,39087^{2} \rightarrow T=279,46 K
\end{gathered}
$$

Fazendo, $M=\frac{u}{c} \rightarrow u=0,39087 * \sqrt{1,4 * 286,9 * 279,46}=130,95 \mathrm{~m} / \mathrm{s}$

De acordo com a Figura 3 as condições iniciais para velocidade no rotor podem ser verificadas utilizando-se da equação em coordenadas cilíndricas e triangulo das velocidades, onde a velocidade rotação é descrita conforme a Equação.

$$
U=\Omega r_{m}
$$

Onde:

$\Omega=$ Velocidade de rotação em $\mathrm{m} / \mathrm{s}$;

$r_{m}=$ Raio médio da linha de centro do Hub para shroud.

Utilizando as relações trigonométricas para o triangulo de velocidades e adotando que a velocidade circunferencial $V_{\theta 1} \cong 0$ e estabelecendo que os resultados utilizados representam "V" velocidades absolutas e "W" velocidades relativas, tem-se:

$$
\begin{gathered}
W_{\theta 1,2}=V_{\theta 1,2}-U_{1,2} \rightarrow V_{\theta 1,2}=0 \therefore W_{\theta 1,2}=-168,82 \mathrm{~m} / \mathrm{s} \\
V_{z 1,2,4}=V_{1,2,4} \operatorname{Cos} \alpha_{1,2,4} \rightarrow V_{1,2,4}=130,9 / \cos -52,20=213,57 \mathrm{~m} / \mathrm{s}
\end{gathered}
$$




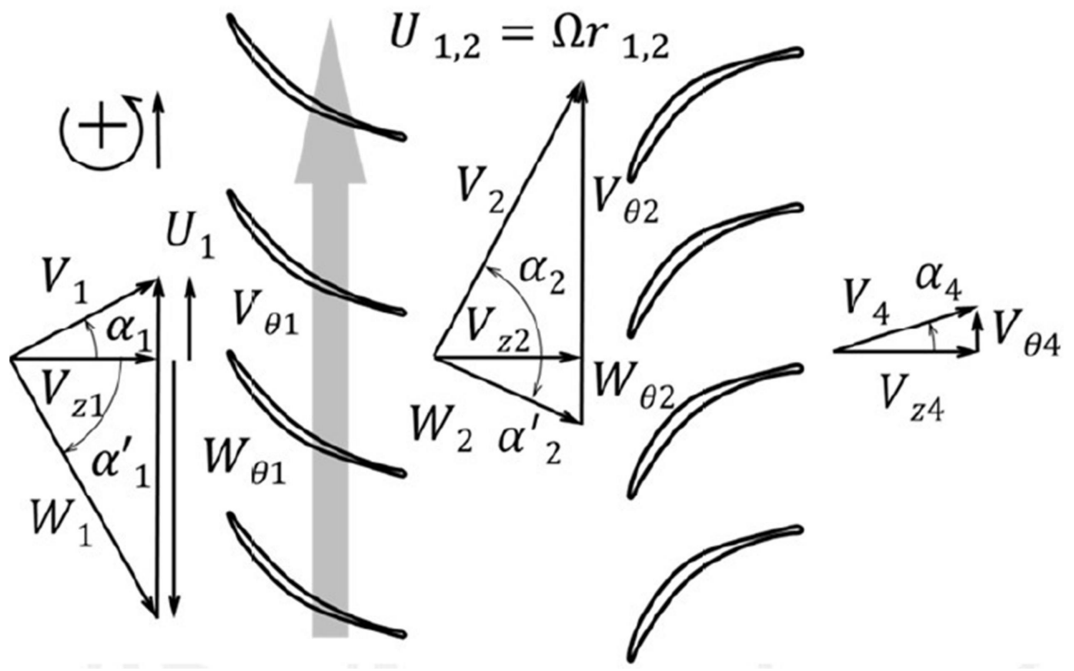

Figura 3: Representação em triangulo das velocidades do escoamento de um fluido entre uma fileira de lâminas rotoras e estatoras.

Tabela 1: Condições de Contorno.

\begin{tabular}{l|l}
\hline Nome & Características \\
\hline Rotor-fluid & $\begin{array}{l}\text { Malha móvel com velocidade angular de 37.500 rpm } \\
\text { na direção-z }\end{array}$ \\
\hline Stator-fluid & Fluido - ar como gás ideal \\
\hline Rotor-blade1 & Wall \\
\hline Rotor-blade2 & Wall \\
\hline Rotor-hub & Wall \\
\hline Rotor-inlet & Pressure-inlet com pressão total de 1 atm e 288 K \\
\hline Rotor-interface & Interface \\
\hline Rotor-per1 & Periódica - faz par com 3 \\
\hline Rotor-per2 & Periódica - faz par com 4 \\
\hline Rotor-per3 & Periódica - faz par com 1 \\
\hline Rotor-per4 & Periódica - faz par com 2 \\
\hline Rotor-shroud & Wall \\
\hline Stator-blade1 & Wall \\
\hline Stator-blade2 & Wall \\
\hline Stator-blade3 & Wall \\
\hline Stator-blade4 & Wall \\
\hline Stator-hub & Wall \\
\hline Stator-interface & Interface \\
\hline Stator-outlet & Pressure-outlet com 1.08 atm e 288 K \\
\hline Stator-per1 & Periódica - faz par com 3 \\
\hline Stator-per2 & Periódica - faz par com 4 \\
\hline Stator-per3 & Periódica - faz par com 1 \\
\hline Stator-per4 & Periódica - faz par com 2 \\
\hline Stator-shroud & Wall \\
\hline &
\end{tabular}




\section{DISCRETIZAÇÃO TEMPORAL}

A solução para malha possui um total de 318.576 elementos e 266.560 Nodes. A Figura 4 apresenta o resultado para malha criada na geometria com tamanho do elemento mínimo de $2 \mathrm{~mm}$ e máximo de $3 \mathrm{~mm}$.
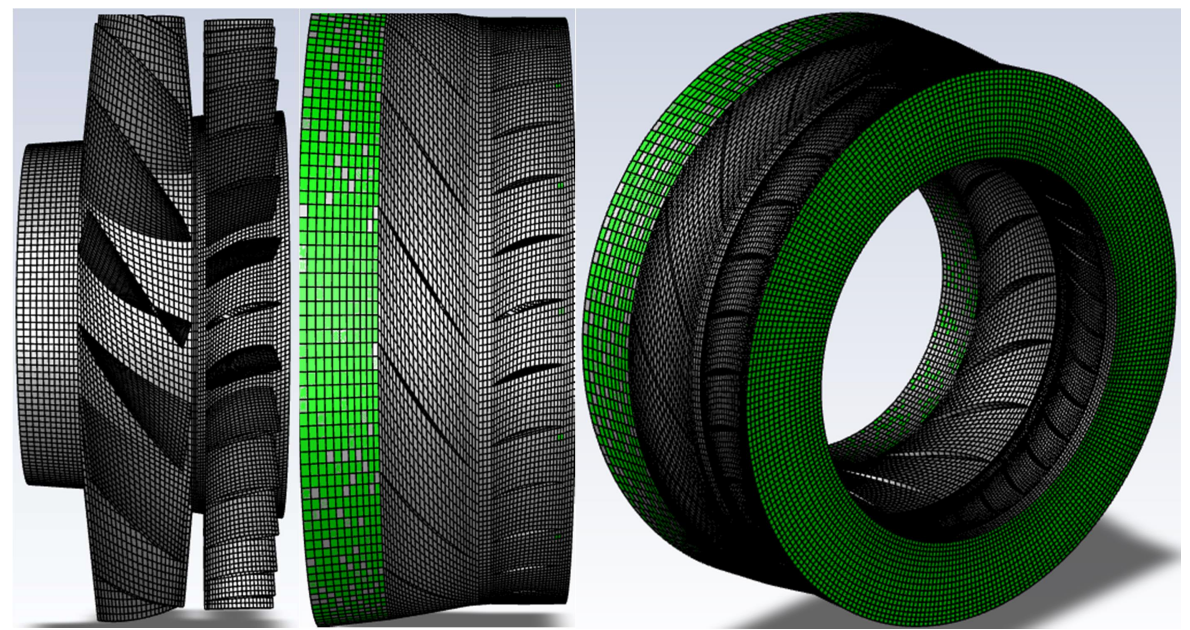

Figura 4: Resultado para malha em toda geometria.

\section{ESTRATEGIA DE SIMULAÇÃO}

O processo de simulação foi executado em três passos, no primeiro, a simulação foi executada utilizando um total de 240 time steps (0,0016 segundos). Esta condição permite avaliar a quantidade de tempo das quais o rotor deverá girar 1.5 graus. Uma vez que o ângulo periódico da lâmina do rotor é de 22.5 graus, o período de passagem da lâmina será de 15 time steps, assim, levando em conta as considerações descritas, o rotor levará um total de 240 time steps para concretizar sua revolução.

Para o segundo passo, utilizou-se mais 720 times steps $(0,0064$ segundos $)$ para solução. Essa condição representa mais três revoluções do rotor gerando um total de 960 time steps. Essa solução deve verificar que a solução é de fato time-periódica.

Após finalizar o segundo passo, para terceira e última solução, adotou-se por mais uma revolução do rotor, ou seja, 240 times steps. Assim, a análise das médias de tempo para a taxa do fluxo de massa nas entradas e saídas durante a revolução final do rotor pode ser verificada. Para isso, utilizou-se da solução Fast Fourier Transform (FFT) para análise da dependência do tempo.

Segundo o manual do solver Fluent R2, a transformada de Fourier lhe permite utilizar qualquer dado dependente de tempo e resolva-o em uma soma equivalente de ondas seno e cosseno. Sendo assim, a interpretação dos dados de sequência de tempo utilizando FFT pode ser melhor avaliada através dos atributos espectrais (frequências) dos dados e para tal, avaliou-se a condição de vazão mássica para entrada e saída, bem como a pressão estática de saída em função da frequência. 
A configuração da simulação utiliza como solução um 3d, Double Precision, DesnsityBased Navier-Stokes Implicit, inviscid e transient Solutions. Além disso, configuração de gás ideal para o ar e esquema de discretização para fluxo de segunda ordem com Least Squares Cell based e transient formulação de primeira ordem. O número de Courant total é de 5 e o time steps é de 6,66666e-06.

\section{RESULTADOS}

Pela física do problema estudado, as variáveis, referente ao estágio do compressor analisado devem apresentar um aumento na pressão estática do rotor / estator, acréscimo na pressão total do rotor e pequena diminuição no estator. Espera-se também aumento da velocidade absoluta no rotor e redução no estator, decréscimo da velocidade relativa no rotor, ao mesmo tempo em que ocorre aumento de densidade do ar em ambos estágios. Consequentemente um aumento da entalpia no rotor, enquanto a entalpia no estator permanece constante.

Através dos gráficos apresentados na Figura 5 é possível verificar que no início da simulação a vazão mássica na entrada e na saída, bem como a pressão estática na saída, possuem instabilidades, ou seja, a solução é transiente no intervalo de 0 a 0,0005 segundos. Observou-se grandes variações para vazão e pressão em ambas as condições de contorno, entrada e saída até o tempo de 0,0005 segundos, porém, a partir de 0,0005 segundos, a solução apresenta convergência e pode ser melhor analisada utilizando-se de mais time steps.

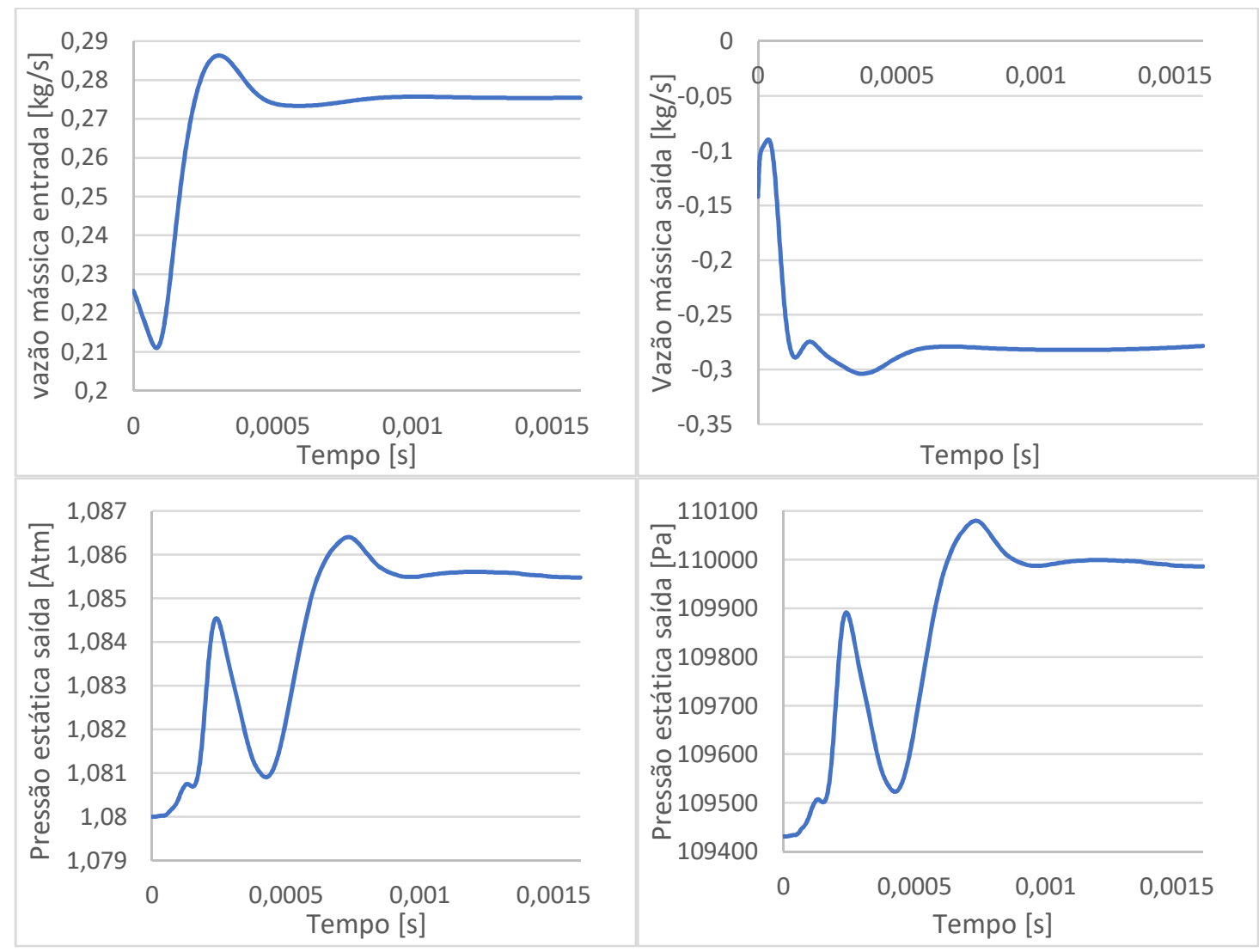

Figura 5: Resultados para vazão mássica e pressão estática utilizando 240 times steps.

Persp. Online: exatas \& eng., Campos dos Goytacazes, 34(11) 44 -58-2021

https://ojs3.perspectivasonline.com.br/ 
A fim de verificar a correta solução do problema, verificou-se o comportamento da vazão mássica na entrada e na saída, bem como a pressão estática na saída e uma escala mais refinada. Através dos gráficos da Figura 6 verifica-se que a solução é de fato time-periódica após o instante de 0,001599 segundos. A periodicidade e observada pelo padrão de repetibilidade das curvas apresentadas. O intervalo de tempo analisado é de 0,0016066 até 0,0064 segundos.

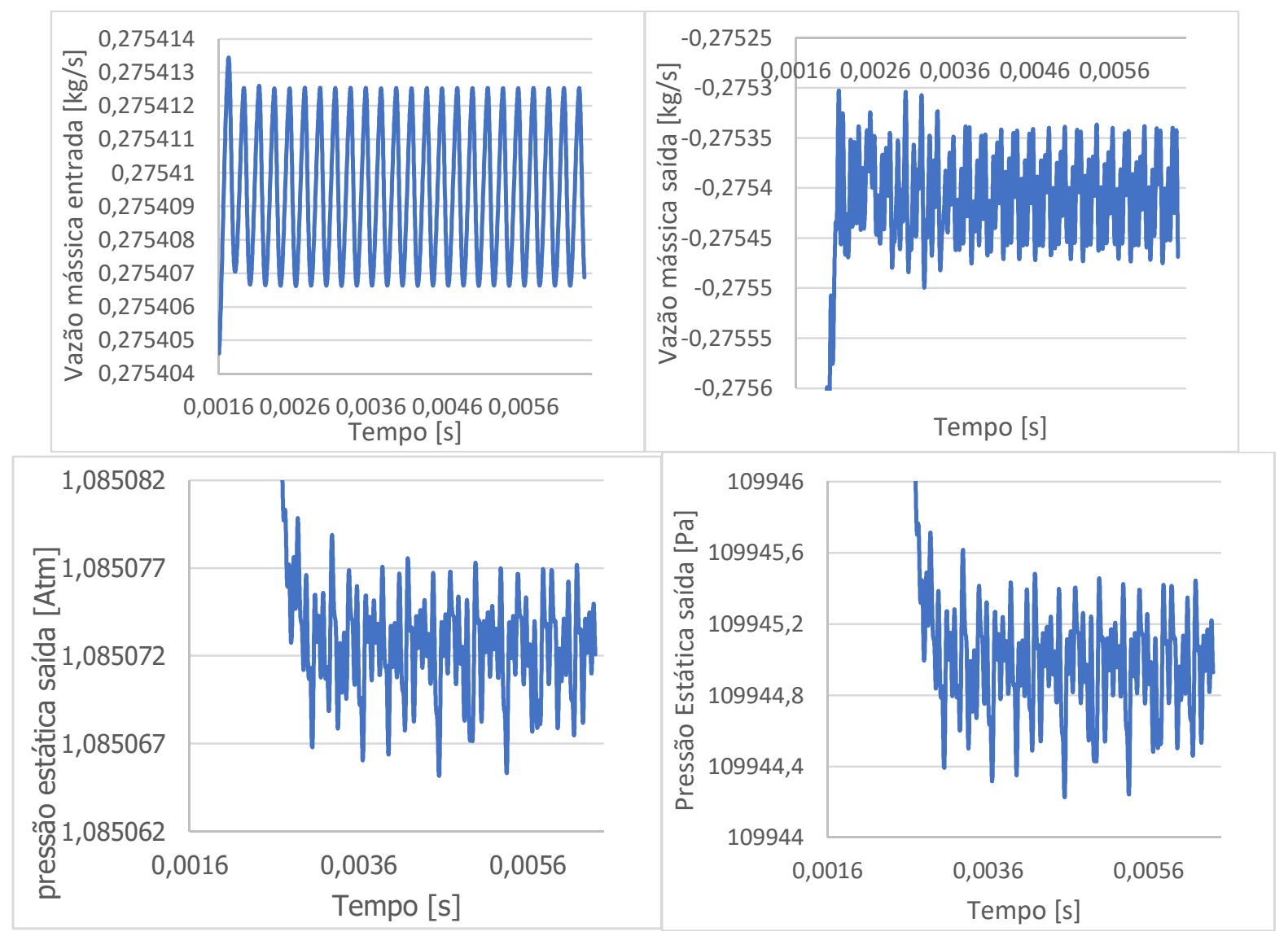

Figura 6: Gráficos obtidos através da solução time-periódica de 241 a 960 time steps.

Para comparação dos resultados analíticos e dos resultados obtidos após simulação, utilizou-se da equação $\dot{m}=\int_{A}(\rho \vec{v} * \hat{n}) d A$. Para uma área de entrada equivalente a $0,001515947 \mathrm{~m}^{2}$, velocidade igual $168,8468 \mathrm{~m} / \mathrm{s}$ e densidade igual $1,081932 \mathrm{~kg} / \mathrm{m}^{3}$ obtém-se o resultado calculado para vazão mássica na entrada que resulta em $0,2769 \mathrm{~kg} / \mathrm{s}$. Utilizando-se da mesma metodologia para saída tem-se, uma área do anular de $0,0009529 \mathrm{~m}^{2}$, velocidade de $229,29 \mathrm{~m} / \mathrm{s}$ e densidade de $1,2568 \mathrm{~kg} / \mathrm{m}^{3}$ obtendo-se $0,2745 \mathrm{~kg} / \mathrm{s}$ para vazão mássica de saída. Todos os resultados quando comparados com a solução computacional apresentam erro de aproximadamente $0,32 \%$ entre as soluções computacional e analítica.

Para verificação da razão de pressão utilizou-se a relação de pressão em função da eficiência isentrópica. A eficiência isentrópica foi verificada através do próprio Fluent 
resultando em 77,5\%. A temperatura de entrada e saída é respectivamente de $288 \mathrm{~K}$ e 332,61K. Resolvendo a Equação (numero), tem-se uma razão de 1,48.

$$
\frac{P_{t}}{P}=\left[1+\frac{0,775 *(332,61-288)}{288}\right]^{\frac{1,4}{1,4-1}}=1,48
$$

Vale ressaltar que a eficiência isentrópica está relacionada ao processo de compressão adiabático e reversível e que a eficiência real do estágio é menor que a eficiência isentrópica. Para o resultado da eficiência isentrópica, este, apresenta-se dentro da faixa de valores obtidos para pesquisa utilizando fluxo supersônico, $75-85 \%$.

Para uma melhor análise do comportamento da vazão e pressão, os resultados obtidos na terceira etapa da simulação foram separados e apresentados nos gráficos conforme mostra a Figura 7. Esta solução apresenta uma configuração diferente das etapas anteriores com a opção de amostras estatísticas para time steps (Fast Fourier Transform, FFT). Este conceito de amostras estatísticas pode ser utilizado para fluxos não estáveis, ou seja, regimes transientes. Com isso, ao habilitar esta opção o Fluent calcula a média de tempo, média dos valores instantâneos, a raiz quadrada média e os erros da raiz quadrada média das quantidades.
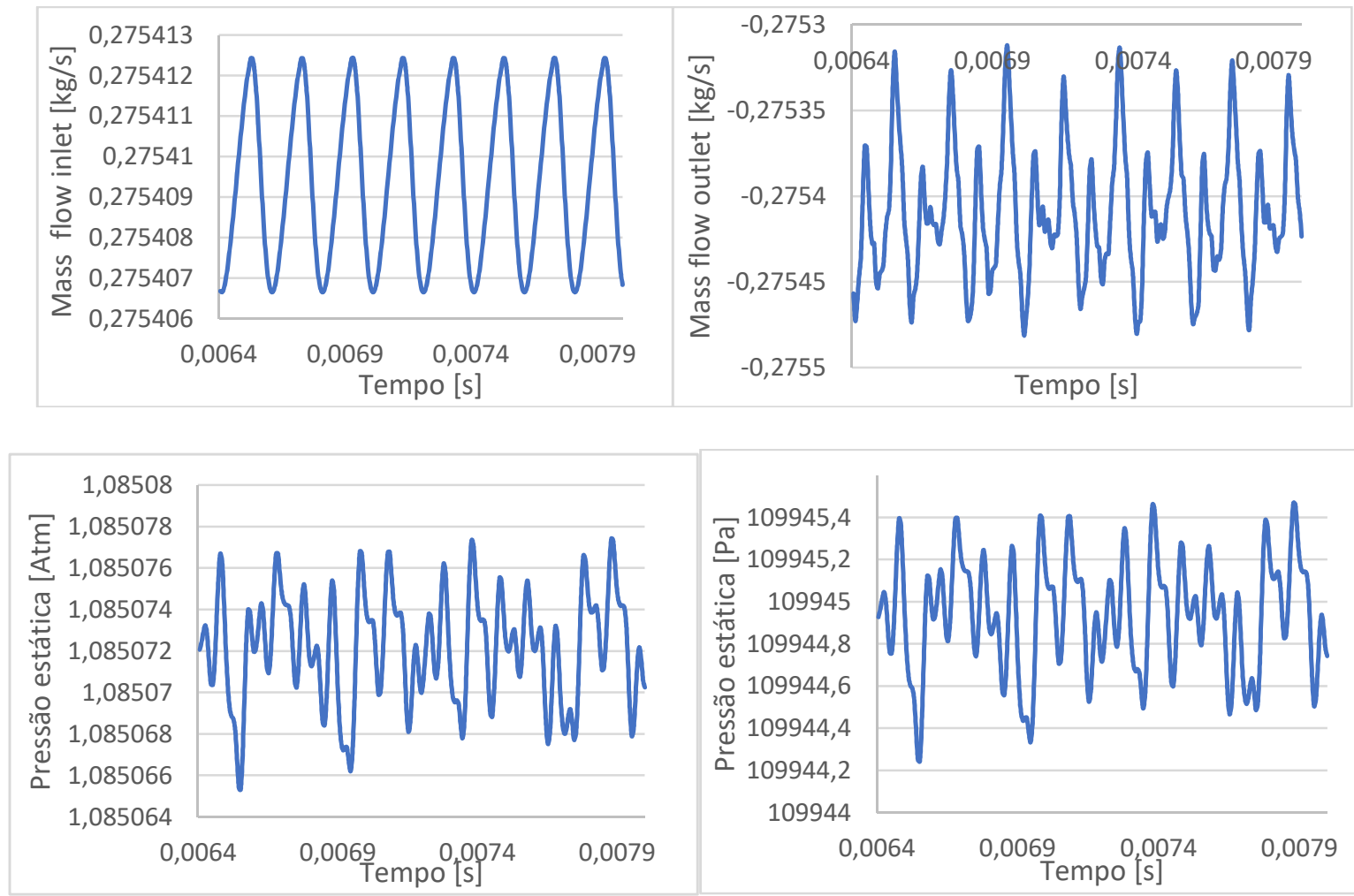

Figura 7: Solução final com 240 time steps.

Comparando os resultados entre os gráficos de entrada e saída para vazão é possível visualizar a conservação de massa através de uma base média de tempo para o sistema ao longo de mais uma revolução. Nota-se que o comportamento das curvas é diferente, a curva

Persp. Online: exatas \& eng., Campos dos Goytacazes, 34(11) 44 -58-2021

https://ojs3.perspectivasonline.com.br/ 
para o rotor na entrada não apresenta o efeito da interação rotor versus estator, enquanto a curva de vazão para saída apresenta o efeito da presença do estator. Os gráficos de pressão também apresentam o efeito da presença do estator.

O resultado de pressão estática é apresentado através da Figura 8. Observa-se que as maiores intensidades de pressão estão localizadas próximos ao bordo de fuga, lado de pressão da lâmina do rotor e no bordo de ataque do estator resultando assim na relação de pressão de 1,086, além disso, ondas de choque são observadas no fluxo próximos a saída do rotor e estator. Estas ondas podem ser verificadas através das alterações de pressão na saída do shroud.

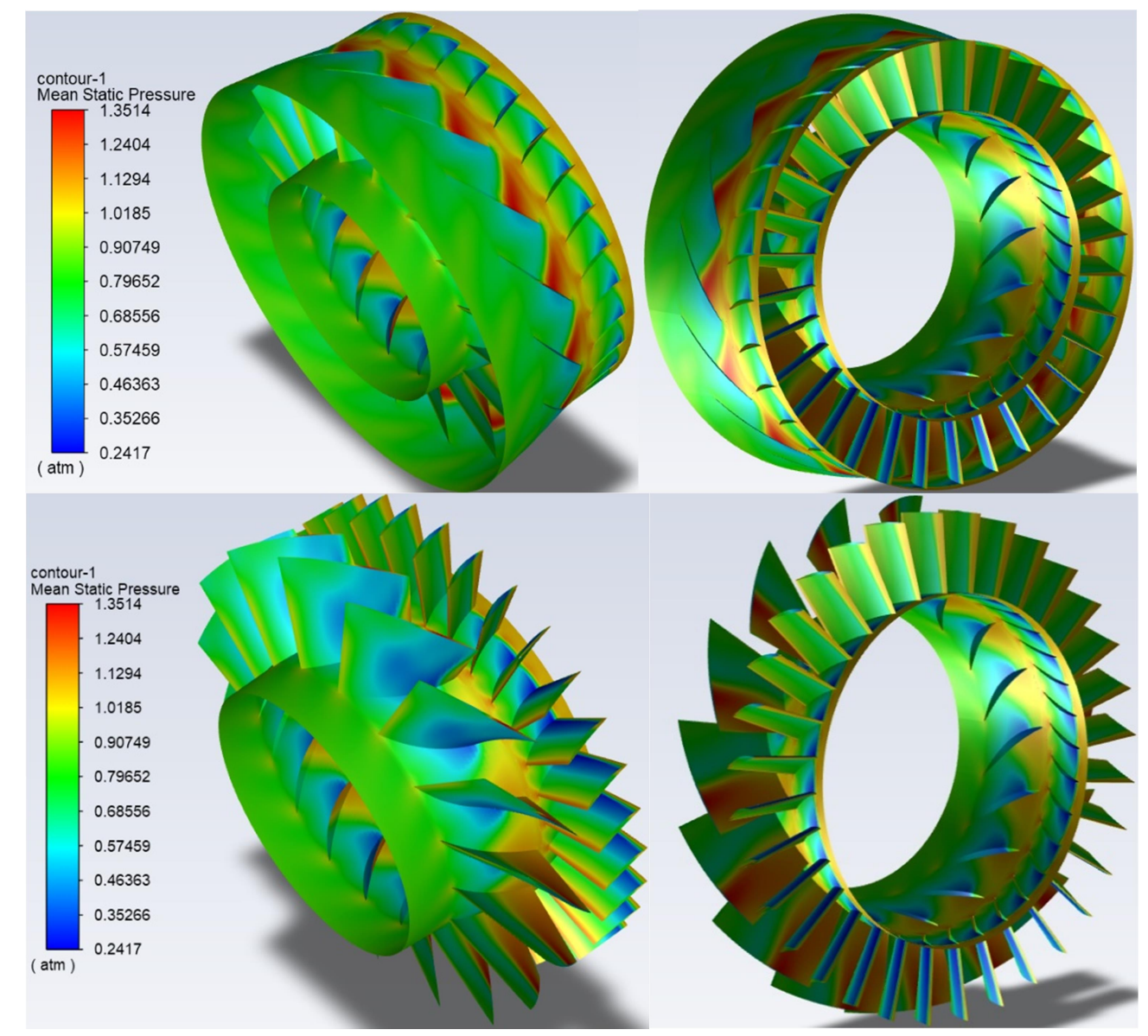

Figura 8: Contorno de pressão estática.

O presente estudo também avaliou o comportamento das principais variáveis envolvidas no estudo do compressor, sendo elas, entalpia, pressão, temperatura, velocidade e densidade do fluido. Os gráficos são valores médios extraídos em regiões especificas e podem ser verificados de acordo com os gráficos referente as Figuras 9 e 10. 


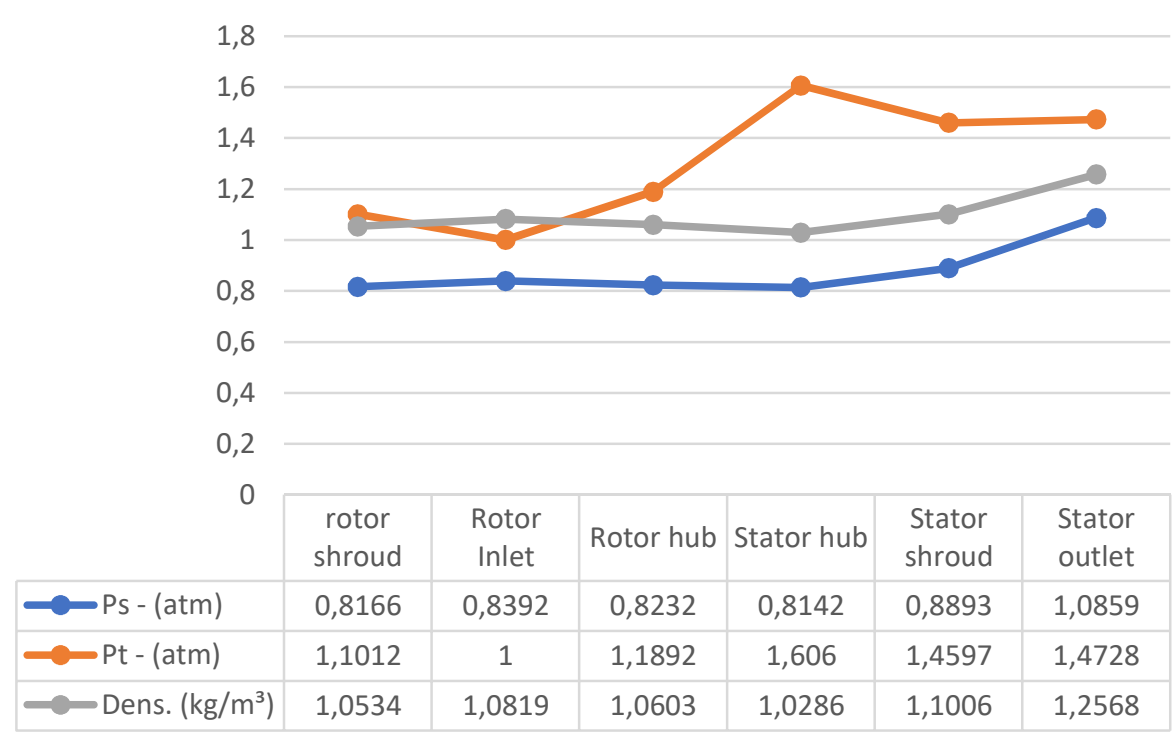

Figura 9: Resultados para pressão estática. pressão total e densidade.

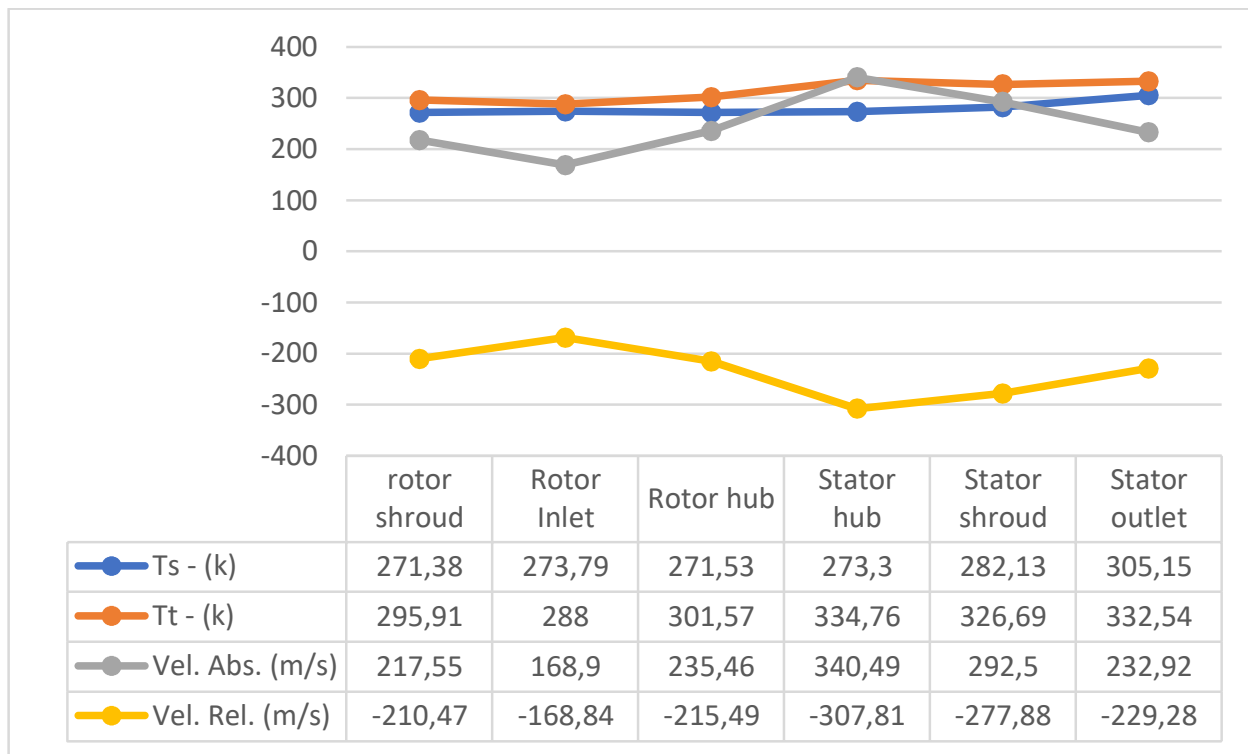

Figura 10: Resultados para temperatura estática, temperatura total, velocidade absoluta e velocidade relativa.

\section{CONCLUSÃO}

O presente trabalho teve como objetivo estudar o comportamento do fluido no primeiro estágio de um compressor axial, assim como estimar a elevação de pressão deste equipamento (máquina), utilizando de ferramentas da dinâmica dos fluidos computacional. Desta forma obteve-se as seguintes conclusões.

- As palhetas rotoras são compostas por um total de 16 lâminas que operam em velocidade de rotação de 37.500 rpm, enquanto as pás do estator somam um total de 32 lâminas.

Persp. Online: exatas \& eng., Campos dos Goytacazes, 34(11) 44 -58-2021

https://ojs3.perspectivasonline.com.br/ 
- O fluido foi tratado como gás ideal, invíscido e com o fluxo periódico, onde o movimento do fluido é direcionado a interagir com todo perfil da pá, resultando em valores mais próximos da realidade.

- A convergência dos resultados foi obtida a partir dos gráficos de resíduos, onde constatouse que a curva apresentou um perfil serrilhado com valores variando de $10^{-1}$ a $10^{-5}$, indicando que o fluxo não é estável, ou seja, o fluido está situado em regime transiente.

- Uma vez que a vazão mássica varia em função das variáveis de operação, constatou-se novamente que a operação se encontra em regime transiente com periodicidade. Corroborado pelo formato com perfil serrilhado.

- Ocorreu um aumento de pressão no primeiro estágio de 1,086 atm, indicando que o perfil das palhetas propostas na simulação não promoveu pressões adversas que possam comprometer a operabilidade do equipamento, porém, foi observado no rotor e na saída do estator descontinuidade de pressão gerando onda de choque. Estas ondas de choque devem ser avaliadas em estudos específicos.

- O processo de aplicação do CFD para o tema proposto, obedece a lei da conservação de massa, onde os valores de entrada e saída devem ser iguais. Isto foi comprovado através dos gráficos de variação da vazão mássica para entrada e saída.

\section{REFERÊNCIAS}

BARJA, G. A Cogeração e Sua Inserção ao Sistema Elétrico. Universidade de Brasília, Brasília, 2006, 171 pp. BRASIL, N., 2005. Notas de Aula de Co-geração do Curso de Engenharia de Equipamentos. Universidade Federal Fluminense, Rio de Janeiro.

ÇENGEL, Y. A., BOLES, M. A. Termodinâmica. 7a ed., McGraw Hill, 2013.

FERZIGER, J. H.; PERIC, M. Computational Methods for Fluid Dynamics. 3.ed. Springer, 2002. 423p.

FORTUNA, A. de O. Técnicas Computacionais para Dinâmica dos Fluidos, Editora da Universidade de São Paulo, 2000.

MARTINELLI, J.L.C. Máquinas Térmicas I: Motores de Combustão Interna. Apostila da UNIJUÍ-Campus Panambi, 2002.

MASCHIETTO, V. C. L. Modelagem das curvas de rendimento de turbinas aeronáuticas turbofan e turbojato. (Monografia) Universidade Tecnológica Federal do Paraná, 2014.

SARAVANAMUTTO, et al. Gas Turbine Theory 6th Edition. Padston, England: Prentice Hall, 2001.

Persp. Online: exatas \& eng., Campos dos Goytacazes, 34(11) 44 -58-2021

https://ojs3.perspectivasonline.com.br/ 
SIMÕES, M. R. Simulação computacional de escoamento turbulento em compressor axial utilizando ferramenta de cfd. (Dissertação) - Universidade Federal do Rio de Janeiro, Rio de Janeiro, 2009.

VALADÃO, Cleuber Pozes. Noções de turbinas a gás. Petrobrás: 2009.

VERSTEEG, H. K.; MALALASEKERA, W. An Introduction to Computation Fluid Dynamics. 2.ed. Pearson Education, 2007. 503p.

WHITE, F M. Mecânica dos Fluidos 6a ed, McGraw Hill, 2011. 